\title{
Ground- and excited-state properties of $M$-center oxygen vacancy aggregates in the bulk and surface of $\mathrm{MgO}$
}

\author{
David Domínguez-Ariza, Carmen Sousa, and Francesc Illas* \\ Departament de Química Física i Centre de Recerca en Química Teòrica, Universitat de Barcelona i Parc Científic de Barcelona, \\ C/Martí i Franquès 1, E-08028 Barcelona, Spain \\ Davide Ricci and Gianfranco Pacchioni \\ Dipartimento di Scienza dei Materiali, Università di Milano-Bicocca, and Istituto Nazionale per la Fisica della Materia, \\ via R. Cozzi, 53-1-20125 Milano, Italy
}

(Received 22 January 2003; published 1 August 2003)

\begin{abstract}
Aggregates of oxygen vacancies ( $F$ centers) represent a particular form of point defects in ionic crystals. In this study we have considered the combination of two oxygen vacancies, the $M$ center, in the bulk and on the surface of $\mathrm{MgO}$ by means of cluster model calculations. Both neutral and charged forms of the defect $M$ and $M^{+}$have been taken into account. The ground state of the $M$ center is characterized by the presence of two doubly occupied impurity levels in the gap of the material; in $M^{+}$centers the highest level is singly occupied. For the ground-state properties we used a gradient corrected density functional theory approach. The dipoleallowed singlet-to-singlet and doublet-to-doublet electronic transitions have been determined by means of explicitly correlated multireference second-order perturbation theory calculations. These have been compared with optical transitions determined with the time-dependent density functional theory formalism. The results show that bulk $M$ and $M^{+}$centers give rise to intense absorptions at about 4.4 and $4.0 \mathrm{eV}$, respectively. Another less intense transition at $1.3 \mathrm{eV}$ has also been found for the $M^{+}$center. On the surface the transitions occur at $1.6 \mathrm{eV}\left(M^{+}\right)$and $2 \mathrm{eV}(M)$. The results are compared with recently reported electron energy loss spectroscopy spectra on $\mathrm{MgO}$ thin films.
\end{abstract}

DOI: $10.1103 /$ PhysRevB.68.054101

PACS number(s): 61.72.Ji, 61.72.Bb, 77.84.Bw

\section{INTRODUCTION}

Oxygen vacancies represent one of the most common point defects in oxides. ${ }^{1}$ Depending on the nature of the material, ionic, covalent, or mixed, the removal of an $\mathrm{O}$ atom from the lattice or from the surface results in a different electronic and geometric rearrangement of the structure. In ionic oxides, such as $\mathrm{MgO}$, the removal of a neutral $\mathrm{O}$ atom from a lattice nominally composed of $\mathrm{O}^{2-}$ and $\mathrm{Mg}^{2+}$ ions results in a cavity (octahedral in the bulk and square pyramidal on the surface) and two electrons. The two electrons are confined in the cavity region by the effect of the strong Madelung field, giving rise to an $F$ center, a point defect with specific optical properties which is responsible for the coloring of the sample. The removal of an $\mathrm{O}^{-}$radical ion, on the other hand, results in a single electron trapped in the cavity, leading to a paramagnetic $F^{+}$color center. $F$ and $F^{+}$centers can be located in the bulk or on the surface of the material (in this latter case the symbol $F_{S}$ is used to distinguish the defect from its bulk counterpart). $F^{+}$centers, being paramagnetic, can be detected by electron paramagnetic resonance (EPR); however, this technique can be applied only to high surface area polycrystalline materials and is of little help for the study of the surface centers on $\mathrm{MgO}$ single crystals or thin films. The other technique which has been widely used to characterize oxygen vacancies is optical spectroscopy.

Bulk $F$ and $F^{+}$centers in $\mathrm{MgO}$ give rise to an intense adsorption band at around $5 \mathrm{eV}$. Kappers et al. ${ }^{2}$ found that the band is actually the convolution of two distinct absorp- tion bands at 4.96 and $5.03 \mathrm{eV}$, due to $F^{+}$and $F$ centers, respectively. Under neutron irradiation other bands at 3.5, 2.1 , and $1.2 \mathrm{eV}$ have been observed ${ }^{3}$ and associated to the presence of aggregates of $F$ centers. The first band is consistent with the appearance of a signal at $3.6 \mathrm{eV}$ upon annealing of $\mathrm{MgO}$ samples containing a high concentration of these point defects and also assigned to $F$-center aggregates. ${ }^{4}$ This is supported by model studies of the kinetics of $F$-center aggregation. ${ }^{5}$ However, while some author has reported similar bands at 3.5 and $2.1 \mathrm{eV}$ in additive coloring experiments, ${ }^{6}$ Chen et al. were not able to observe these transitions in electron irradiated additive colored samples. ${ }^{3} \mathrm{X}$ ray $^{7}$ and UV (Ref. 8) irradiation results in a band around $2 \mathrm{eV}$ but does not give rise to features around $3.5 \mathrm{eV}$. Therefore, while it is generally accepted that the intense band at $5 \mathrm{eV}$ originates from bulk $F$ and $F^{+}$centers, the assignment of the band at $3.5 \mathrm{eV}$ to aggregates of $F$ centers is not unambiguous, and no proposals seem to exist for the band around $2 \mathrm{eV}$.

The detection of surface $F$ centers is even more difficult because of the problems connected to surface sensitivity of the measure. By creating surface defects on $\mathrm{MgO}$ by various techniques one observes transitions in a wide range, from 1 to $5 \mathrm{eV} ; ;^{9-12}$ see Table I. The bands at $5 \mathrm{eV}$ have been attributed to subsurface $F$ centers, while the bands in the $2-3 \mathrm{eV}$ region were assigned tentatively to surface $F$ (Ref. 9) or $V^{-}$ (Ref. 10) centers. Wu et al. ${ }^{11}$ did perform the first experiment on $\mathrm{MgO}$ thin films and did not observe bands in the $2-3 \mathrm{eV}$ region, but one band at 1.15 and $3.6 \mathrm{eV}$. These bands were tentatively assigned to surface $F$ centers $(1.15 \mathrm{eV})$ and to $F$ centers aggregates $(3.6 \mathrm{eV})$, respectively. In a recent paper 
TABLE I. Summary of experimental results on optical transitions in $\mathrm{MgO}$ bulk and surface.

\begin{tabular}{ccc}
\hline \hline Generation of defects & Character of the sample & \multicolumn{1}{c}{ Transitions observed } \\
\hline X-ray irradiation & & $2.4,4.4,5.6 \mathrm{eV}$ (Ref. 7) \\
UV irradiation & Single crystal, bulk & $1.2,2.1,4.8 \mathrm{eV}$ (Ref. 8) \\
Neutron irradiation & & $1.2,2.1,3.5,4.8 \mathrm{eV}$ (Refs. 3, 8) \\
Additively colored crystals & & $0.9,1.1,4.8 \mathrm{eV}$ (Ref. 3) \\
(Mg excess) & & $2.1,3.7,4.8 \mathrm{eV}$ (Ref. 6) \\
Electron irradiation & & $4.8 \mathrm{eV}$ (Ref. 3) \\
Electron irradiation & Single crystal, surface & $2.3 \mathrm{eV}$ (Refs. 9, 10) \\
& $\mathrm{MgO}$ thin film on Ag(100) & $1.0,1.3,2.4,2.8,3.4 \mathrm{eV}$ (Ref. 12) \\
Thermal treatment & $\mathrm{MgO}$ thin film on $\mathrm{Mo}(100)$ & $1.2,3.6,5.3 \mathrm{eV}$ (Ref. 11) \\
\hline \hline
\end{tabular}

by Pfnür and co-workers, ${ }^{12} \mathrm{MgO}$ thin films have been electron bombarded and the corresponding optical properties have been measured by electron energy loss spectroscopy (EELS). These authors tentatively assign the transitions at around $1 \mathrm{eV}$ to surface $M$ centers, i.e., an aggregate of two adjacent $F$ centers. Likewise, on the basis of the comparison with $a b$ initio calculations of the optical properties of these centers, ${ }^{13,14}$ the bands at $2.4,2.8$, and $3.4 \mathrm{eV}$ have been attributed to the presence of surface $F$ centers at different coordinated sites. Given the uncertainty in the proposed assignments, it is clear that a comparison of measured with computed transition energies can be of great help.

While the ground state properties of $F$ centers have been studied in some detail from a theoretical point of view, ${ }^{15-19}$ few $a b$ initio calculations have been reported on the excited states of $F$ centers, or on the ground and excited state properties of $F$ centers aggregates (e.g., the $M$ center). Finocchi et $a l^{20}$ have studied the interaction of surface oxygen vacancies on the $\mathrm{MgO}(100)$ surface by means of periodic calculations in the framework of the density functional theory (DFT) within the local density approximation (LDA). They found that, at low defect concentration, a series of electronic levels is created in the gap of the $\mathrm{MgO}$ surface. These levels are fully occupied and result from the coupling of atomiclike orbitals localized on the vacancy sites. At high defect concentrations a mixing between these levels and the conduction band levels occurs. Miyoshi et al. ${ }^{21}$ have studied the excitation energies for bulk and surface $F$ centers and for bulk $M$ centers using the Hartree Fock (HF) approach followed by a rather limited multiconfigurational self-consistent field (MC$\mathrm{SCF})$ treatment. Their prediction for the excitation energy of bulk $F$ and $M$ centers, 7.38 and $5.79 \mathrm{eV}$ respectively, are much too high. The main source of error in these results is the lack of electronic correlation effects in the calculated wave functions. Recently we have studied the optical properties of $F$ centers in the bulk and on different sites of the $\mathrm{MgO}(100)$ surface, using explicitly correlated wave functions, ${ }^{13,14}$ and we found an excitation energy for the bulk $F$ and $F^{+}$centers of around $6 \mathrm{eV}$ instead of $5 \mathrm{eV}$, as experimentally observed. The error is largely due to limitations in the size of the basis set and of the cluster used, as shown in a successive study. ${ }^{22}$ Still, assuming a given overestimate of about $15 \%$ in the computed excitation energies, it has been possible to predict that surface $F$ centers give rise to transi- tions in the $2-3 \mathrm{eV}$ region depending on their location on the surface. However, no absorption band was predicted below 2 $\mathrm{eV}$, leaving open the problem of the assignment of this band.

In this work, we report the first $a b$ initio study of the transition energies of a pair of $F$ centers, the $M$ center, in the bulk and on the surface of $\mathrm{MgO}$. For comparison, $F$ centers have also been considered to provide an error bar to the computed optical transitions.

\section{COMPUTATIONAL APPROACH}

Optical transitions for surface and bulk $F$ and $M$ centers have been studied using the complete active space selfconsistent field (CASSCF) and CAS second-order perturbation theory (CASPT2) methods ${ }^{23,24}$ applied to suitably embedded cluster models. Likewise, time-dependent (TD) density functional theory ${ }^{25}$ (DFT) calculations have also been performed mainly to explore the performance of this rather new methodology in this particular kind of systems. For molecular systems, TD-DFT is known to provide a good accuracy ${ }^{26-28}$ while relatively few examples of applications to optical transitions of defects in solids have been reported. ${ }^{29}$

CASPT2 is a generalization to CASSCF wave functions of the well-known second-order Møller-Plesset perturbation scheme (MP2) based on closed-shell HF reference wave functions, and reduces rigorously to MP2 for CAS containing a single closed-shell Slater determinant. An important part of the electron correlation effects is treated in a variational way in the CASSCF step, and the remainder, mainly dynamical electron correlation, is estimated by second-order perturbation theory with the CASSCF as zeroth-order wave function. This strategy combines the accuracy of a multireference configuration interaction treatment and the low computational cost of a perturbational approach. Over the last few years the CASPT2 method has been proven to be a fruitful approach to study, analyze, and predict the spectroscopy of a wide range of organic and inorganic molecules. ${ }^{30-33}$ The method has also been successfully applied to study excited states in solid state compounds. ${ }^{34-37}$ For $F$ centers, the active space used includes the two vacancy orbitals involved in the electronic transition and 2 or 1 electrons for $F$ and $F^{+}$centers, respectively. For $M$ centers, the active space includes four orbitals for each vacancy (one of $s$ type and the three 
components of one $p$-type orbital). Therefore, the CAS contains eight orbitals and four (or three) electrons for $M$ (or $M^{+}$) centers. The CASSCF/CASPT2 calculations have been performed using the MOLCAS 5 package. ${ }^{38}$

TD-DFT is based in the Kohn-Sham formulation of DFT and makes use of the eigenvalues and eigenvectors of the Kohn-Sham equations. In this formalism, the exact nonlocal HF exchange for a single determinant is replaced by a general expression, the exchange correlation functional, which in principle includes both exchange and electron correlation energy terms. Here we have chosen the hybrid B3LYP approach where the HF exchange is mixed in with the DFT exchange using the Becke three-parameter approach ${ }^{39}$ combined with the nonlocal expression of the correlation functional proposed by Lee, Yang, and Parr, ${ }^{40}$ based on the original work of Colle and Salvetti on the correlation factor. ${ }^{41,42}$

Two strategies have been followed to embed the quantummechanical (QM) clusters depending on the theoretical method used. For CASPT2 calculations we used clusters of ions embedded in point charges (PC's). An interface of $a b$ initio model potentials (AIMP's) (Refs. 43-45) between the PC's and the cluster has been used to avoid an artificial polarization of the anions electronic density induced by the PC's. For DFT calculations long-range polarization effects have also been taken into account. To this end, the $\mathrm{MgO}$ cluster model is divided into regions I and II. Region I includes a QM treated cluster, which is exactly the same as in the CASPT2 calculations, but surrounded by interface ions and classical shell model ions. ${ }^{46,47}$ The remaining part of the cluster, region II, is represented by PC's. The interface between the QM cluster and the classical ions, needed to prevent an artificial spreading of electronic states outside the QM cluster, is based on the representation of the $\mathrm{Mg}^{2+}$ ions by a semilocal effective core pseudopotential (ECP). ${ }^{48}$ All the classical ions interact among themselves via interatomic potentials. The interface atoms interact quantum mechanically with the QM cluster, and classically with the remaining "atoms" in regions I and II. The interaction between the QM atoms and classical ions in region I is described using shortrange classical potentials and long-range Coulomb potentials whereas interaction with atoms in regions II includes only the long-range potential. All centers in region I are allowed to relax simultaneously during the geometry optimization. PC's in region II remain fixed and provide an accurate electrostatic potential within region I. This hybrid scheme is implemented in the GUESS code, ${ }^{46}$ which provides the shellmodel representation for the classically treated part of the system and an interface with the GAUSSIAN98 package for $a b$ initio calculations ${ }^{49}$ of the $\mathrm{QM}$ cluster. The GUESS code allows us to calculate forces acting on all centers in region I, both QM and classical (cores and shells) and simultaneously optimize their positions using the Broyden-FletcherGoldfarb-Shanno technique ${ }^{50}$ for the energy minimization. We denote these clusters as shell-model (SM) embedded clusters. Ground state geometry optimizations have been performed at the B3LYP level using the SM-embedded clusters. The CASPT2 excitation energies have been computed on these optimal geometries. The excitation energies of the $F$ and $M$ centers have also been computed using the time- dependent density functional theory approach (TD-DFT) within the B3LYP functional.

At the CASPT2 level three clusters of different shape and size have been used for bulk $F$ centers: $\mathrm{Mg}_{6} \mathrm{O}_{18}(\mathrm{QM})$ $+\mathrm{Mg}^{2+}{ }_{159} \mathrm{O}^{2-}{ }_{159} \quad$ (AIMP's), $\quad \mathrm{Mg}_{14} \mathrm{O}_{12}(\mathrm{QM})$ $+\mathrm{Mg}^{2+}{ }_{157} \mathrm{O}^{2-}{ }_{159}$ (AIMP's), and $\mathrm{Mg}_{14} \mathrm{O}_{18}(\mathrm{QM})$ $+\mathrm{Mg}^{2+}{ }_{157} \mathrm{O}^{2-}{ }_{153}$ (AIMP's). For bulk $M$ centers two different clusters have been used: $\mathrm{Mg}_{10} \mathrm{O}_{26}(\mathrm{QM})+\mathrm{Mg}^{2+}{ }_{88} \mathrm{O}^{2-}{ }_{62}$ (AIMP's) and $\mathrm{Mg}_{24} \mathrm{O}_{22}(\mathrm{QM})+\mathrm{Mg}^{2+}{ }_{66} \mathrm{O}^{2-}{ }_{66}$ (AIMP's). For surface $M$ centers also two clusters have been used: $\mathrm{Mg}_{8} \mathrm{O}_{18}(\mathrm{QM})+\mathrm{Mg}^{2+}{ }_{52} \mathrm{O}^{2-}{ }_{34}$ (AIMP's) and $\mathrm{Mg}_{16} \mathrm{O}_{14}(\mathrm{QM})$ $+\mathrm{Mg}^{2+}{ }_{38} \mathrm{O}^{2-}{ }_{38}$ (AIMP's). At DFT level one cluster has been used for bulk $M$ centers, composed of a $\mathrm{Mg}_{10} \mathrm{O}_{26}$ quantum-mechanical part, $50 \mathrm{Mg}^{2+}$ ECP's and 902 polarizable shells. For surface $M$ centers the cluster used consisted of a $\mathrm{Mg}_{16} \mathrm{O}_{14}$ quantum-mechanical part, $24 \mathrm{Mg}^{2+}$ ECP's and 782 polarizable shells. All these clusters were embedded in a large array of \pm 2 PC's.

Different basis sets have been used in the CASPT2 and DFT calculations. This is due to the slower convergence towards basis set limit of the explicitly correlated calculations compared to DFT calculations. For DFT calculations, a 6-31 $\mathrm{G}$ basis set has been used on all $\mathrm{Mg}^{51}$ and $\mathrm{O}^{52}$ atoms in the geometry optimization. For the computation of the excitation energies an oxygen $6-31+G^{*}$ basis set ${ }^{53,54}$ has been added in the center of the vacancies to improve the description of the localized levels. For CASSCF/CASPT2 calculations atomic natural orbitals (ANO) basis sets for $\mathrm{O}^{55}$ and $\mathrm{Mg}^{56}$ atoms with different contraction schemes have been used. The contraction scheme for the smallest basis set used (basis A) is [ $14 s 9 p 4 d / 3 s 2 p 1 d]$ for $\mathrm{O}$ and $[17 s 12 p / 3 s 2 p]$ for $\mathrm{Mg}$. For the second basis set (basis B), the contraction scheme is $[14 s 9 p 4 d / 4 s 3 p 1 d]$ for $\mathrm{O}$ and $[17 s 12 p / 4 s 3 p]$ for $\mathrm{Mg}$. A third basis set (basis $C$ ) has been used for $F$ centers, in which a $[5 d / 1 d]$ basis function has been added to the basis set of the six Mg atoms surrounding the vacancy. Finally, a fourth basis set has been used (basis D) for bulk $M$ centers, this is $[14 s 9 p 4 d / 4 s 3 p 1 d]$ for $\mathrm{O}$ and $[17 s 12 p / 4 s 3 p]$ for the ten $\mathrm{Mg}$ surrounding the cavity and $[17 s 12 p / 3 s 2 p]$ for the remaining $\mathrm{Mg}$ atoms in the cluster. In all cases, a $(3 s 2 p 1 d)$ uncontracted basis set has been placed in the center of the vacancies in order to improve the description of the electronic states. These functions have been optimized for the ground state of the $F^{+}$center at the HF level in a previous work. ${ }^{13}$

\section{RESULTS}

\section{A. Bulk $F$ centers}

Before discussing the results for the $M$ centers, we consider the bulk $F$ and $F^{+}$centers of $\mathrm{MgO}$ where unambiguous assignments of the corresponding transition energies can be done. In this way we want to check the accuracy of the methods used and the convergence of the results versus cluster size and basis set quality. The ground state of the $F$ center is characterized by the presence of a doubly occupied electronic level in the mid of the band gap. This level belongs to the total symmetric $A_{1 g}$ representation in $O_{h}$ symmetry thus 


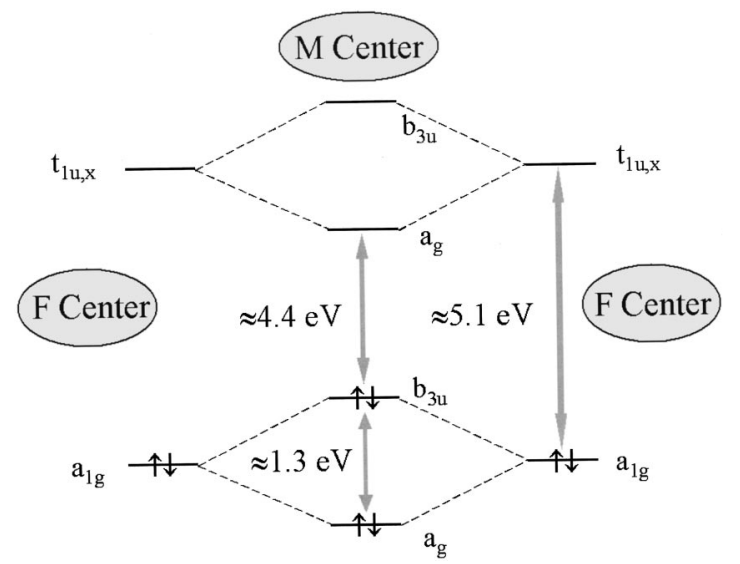

FIG. 1. Schematic representation of the energy levels involved in the electronic transitions of bulk $F$ and $M$ centers. The energies reported refer to the computed excitations energies for the bulk centers at the CASPT2 level.

leading to a ${ }^{1} A_{1 g}$ electronic state arising from the $\left(a_{1 g}\right)^{2}$ electronic configuration. The ground state of the $F^{+}$center is a ${ }^{2} A_{1 g}\left(a_{1 g}\right)^{1}$ in which the $a_{1 g}$ vacancy level is singly occupied. The first empty level of the bulk $F$ center is of " $p$ " nature and, due to the $O_{h}$ symmetry, is threefold degenerate $\left(t_{1 u}\right)$. The first optical transition is, therefore, an excitation from the $a_{1 g}$ level to the $t_{1 u}$ one $\left(a_{1 g} \rightarrow t_{1 u}\right)$ for both the $F$ and the $F^{+}$centers, see Fig. 1.

Since the scope of this work is to study the allowed optical transitions, we restrict the discussion to singlet and doublet excited states for $F$ and $F^{+}$centers, respectively. The results of Table II have been obtained using the $\mathrm{Mg}_{14} \mathrm{O}_{12}$ cluster at the experimental bulk geometry and allow us to define the adequacy of the basis set used in CASSCF and CASPT2 calculations. In general we observe that the CASSCF method gives consistently too high transition energies; this is not surprising since CASSCF does not include dynamical correlation. The inclusion of dynamical correlation at CASPT2 level lowers the transition energies by up to $1 \mathrm{eV}$. Therefore, in the following we restrict the discussion to the CASPT2 results. With basis set B the excitation energy is of $5.40 \mathrm{eV}$ for $F$ and of $5.90 \mathrm{eV}$ for $F^{+}$. These results are in line with our previous work. ${ }^{22}$ The use of the large basis set $\mathrm{C}$ has only a modest effect on the excitation energy of the $F$ center, suggesting that the results are reasonably converged versus basis set size; even going from basis A to basis B the changes are rather small $(<0.1 \mathrm{eV})$. Both transitions energies are overestimated, especially for the $F^{+}$center. The limited inclusion of electron correlation and the use of an unrelaxed bulk geometry are likely to be the reasons for this overestimate, as will be discussed below. Consequently, in the calculation of the $M$ centers we will adopt these two basis sets (A and B) which seem to provide a good compromise between accuracy and computational cost. Next, the dependence of the results versus cluster size and shape for both $F$ and $F^{+}$centers have been investigated always using an unrelaxed bulk geometry and basis A, Table III. Three clusters have been used: $\mathrm{Mg}_{6} \mathrm{O}_{18}, \mathrm{Mg}_{14} \mathrm{O}_{12}$, and $\mathrm{Mg}_{14} \mathrm{O}_{18}$. Going from the smallest $\mathrm{Mg}_{6} \mathrm{O}_{18}$ cluster to $\mathrm{Mg}_{14} \mathrm{O}_{12}$ a reduction of the first singlet-to-singlet transition for the $F$ center from 5.96 to $5.47 \mathrm{eV}$ is observed. An additional increase of cluster size, $\mathrm{Mg}_{14} \mathrm{O}_{18}$, has only minor effects on this transition energy which becomes $5.55 \mathrm{eV}$. The dependence of the transition energy on cluster size seems to be less pronounced for the $\mathrm{F}^{+}$center because of the more localized nature of the excited state. Therefore, results in Table III suggest that a QM cluster of 20-30 atoms is sufficient to describe the main features of the excited states of $F$ centers. Clearly, what remains to be checked is the effect of the geometry relaxation on the excitation energies. An optimization of the geometry for the $F$ and $F^{+}$centers has been performed at the HF level with a SM-embedded cluster $\left(\mathrm{Mg}_{14} \mathrm{O}_{18}\right.$ embedded in 30 ECP's, 956 polarizable ions, and 3450 PC's). For the $F$ center, the distance between the vacancy and the closest $\mathrm{Mg}$ ions expands by $3.2 \%$ with respect to the $\mathrm{Mg}-\mathrm{O}$ bulk distance. For $F^{+}$the expansion increases to $7.4 \%$. The relaxation of the geometry has a significant effect on the computed excitation energies. These have been determined for the $\mathrm{Mg}_{14} \mathrm{O}_{12}$ cluster using basis $\mathrm{C}$ and the CASPT2 approach, Table III. The computed transition energies are $5.01 \mathrm{eV}$ for $F$ and 5.22 $\mathrm{eV}$ for $F^{+}$centers, respectively. This means that the error in the computed excitation energies is roughly $5 \%$ for $F^{+}$center and less than $1 \%$ for the $F$ center. Thus, not only the fact that $F$ and $F^{+}$centers give rise to a similar transition energy is reproduced, but even the absolute value of the transition is in quantitative agreement with experiment.

Analogous calculations for the ground and excited states of $F$ and $F^{+}$centers have been carried out using the TD-DFT formalism within the B3LYP functional. However, a number of problems in the determination of the excited state energies have been encountered which resulted in much too low cal-

TABLE II. CASSCF/CASPT2 excitation energies (in eV) for $F$ and $F^{+}$bulk centers using the cluster $\mathrm{Mg}_{14} \mathrm{O}_{12}$ at the experimental bulk geometry and different basis sets. Only spin allowed transitions are reported. Symmetry of the electronic states involved is indicated.

\begin{tabular}{lcccccc}
\hline \hline & \multicolumn{2}{c}{ Basis A } & \multicolumn{2}{c}{ Basis B } & \multicolumn{2}{c}{ Basis C } \\
& CASSCF & CASPT2 & CASSCF & CASPT2 & CASSCF & CASPT2 \\
\hline $\begin{array}{l}\text { Bulk } F \\
{ }^{1} A_{1 g} \rightarrow{ }^{1} T_{1 u}\end{array}$ & 6.45 & 5.47 & 6.34 & 5.40 & 6.27 & 5.33 \\
\hline $\begin{array}{l}\text { Bulk } F^{+} \\
{ }^{2} A_{1 g} \rightarrow{ }^{2} T_{1 u}\end{array}$ & 6.73 & 5.96 & 6.65 & 5.90 & 6.66 & 5.84 \\
\hline \hline
\end{tabular}


TABLE III. CASPT2 excitation energies (in eV) for $F$ and $F^{+}$bulk centers using different cluster models, experimental bulk geometry and basis set A. Only spin allowed transitions are reported. Symmetry of the electronic states involved is indicated.

\begin{tabular}{|c|c|c|c|c|c|}
\hline & $\mathrm{Mg}_{6} \mathrm{O}_{18}$ & & & $\mathrm{Mg}_{14} \mathrm{O}_{18}$ & Exp. assignment \\
\hline${ }^{1} A_{1 g} \rightarrow{ }^{1} T_{1 u}$ & 5.96 & 5.47 & $5.01^{\mathrm{a}}$ & 5.55 & 5.03 (Ref. 2) \\
\hline $\begin{array}{l}\text { Bulk } F^{+} \\
{ }^{2} A_{1 g} \rightarrow{ }^{2} T_{1 u} \\
\end{array}$ & 6.00 & 5.96 & $5.22^{\mathrm{a}}$ & 5.95 & 4.96 (Ref. 2) \\
\hline
\end{tabular}

${ }^{a}$ Results obtained on a fully optimized geometry with basis set C.

culated values for the optical transitions or in convergence problems. Here, we can only attempt a tentative explanation for this behavior and plan to perform more extended analyses of the problem in a specific study. One may think that the failure of the TD-DFT is associated to the well-known feature of DFT methods to underestimate band gaps. However, this is not the case because the present TD-DFT calculations have been carried out using the hybrid B3LYP functional which indeed properly describes the band gap of oxides. ${ }^{57,58}$ The analysis of the TD-DFT results shows that the TD-DFT excited states of bulk $F$ and $F^{+}$centers have an excessive conduction band character. This mixing with conduction band orbitals in the $F$ and $F^{+}$excited states is due to the combination of two factors. On the one hand, the intrinsic monoelectronic character of TD-DFT which makes use of the Kohn-Sham eigenvalues of the ground state and, on the other hand, the strong dependence of the energy of virtual orbitals on cluster size. It has been shown that the band gap estimate of $\mathrm{MgO}$ by means of a cluster model is rather cluster size dependent. ${ }^{59}$ In other words, because of the abovementioned problems TD-DFT describes a genuine singlereference excitation as a multireference one. CASPT2 does not suffer from this defficiency because the energy of the excited state is computed directly from the $N$-electron wave function and both, the ground and excited state wave functions, have a marked single reference character.

\section{B. Bulk $M$ and $M^{+}$centers}

$M$ centers are formed by removing two adjacent $\mathrm{O}$ atoms thus forming a double cavity. The electronic structure of a bulk $M$ center is characterized by the presence of two electronic levels in the gap which belong to the $A_{g}$ and $B_{3 u}$ irreducible representations in $D_{2 h}$. These levels arise from the coupling of the occupied $a_{1 g}$ levels of the isolated $F$ centers, Fig. 1, which results in a bonding $a_{g}$ and an antibonding $b_{3 u}$ orbital [Figs. 2(a) and 2(b)]. In the ground state of neutral $M$ centers both levels are doubly occupied-

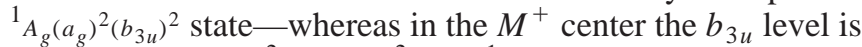
a singly occupied ${ }^{2} B_{3 u}\left(a_{g}\right)^{2}\left(b_{3 u}\right)^{1}$ state. The coupling of the $t_{1 u, x}$ empty levels of the isolated $F$ centers forms the excited levels of $M$ centers of $A_{g}$ symmetry, Fig. 2(c). This is the level involved in the lowest transitions of the $M$ center. The $x$ axis is assumed to pass through the center of the vacancies so that the first dipole allowed transition of the $M$ center corresponds to the excitation of one electron from the filled $b_{3 u}$ orbital to the virtual $a_{g}$ one $\left(b_{3 u} \rightarrow a_{g}\right)$, see Fig. 1. For $M^{+}$, in addition to this transition, there is a second excitation which corresponds to the transfer of one electron from the doubly occupied $a_{g}$ orbital to the singly occupied $b_{3 u}$ level $\left(a_{g} \rightarrow b_{3 u}\right)$, Fig. 1. We will see below that this second transition is the lowest one in $M^{+}$centers. This description of the electronic levels of the $M$ centers is analogous to that reported by Miyoshi et al. ${ }^{21}$ for bulk $M$ centers in $\mathrm{MgO}$, Kölmel and Ewig ${ }^{60}$ for bulk $M$ centers in LiF, and by Finocchi et $a .^{20}{ }^{20}$ for $\mathrm{MgO}$ surface $M$ centers. Since we are interested in allowed optical transitions, we discuss only the results for the singlet and doublet excited states of $M$ and $M^{+}$ centers, respectively.

The geometry optimization for the ground state performed at the DFT (B3LYP) level leads to a small relaxation for the neutral $M$ center, with an average expansion of the distances between the center of the vacancy and the nearest magnesium atoms of $1.54 \%$. For the $M^{+}$center the relaxation is larger, and the average expansion of the distance of the $\mathrm{Mg}$ ions from the center of the cavity is of $4.12 \%$. Geometry relaxation has a small impact on the computed excitation energies: the $b_{3 u} \rightarrow a_{g}$ transition for the $M$ center changes by about $0.2 \mathrm{eV}$, while the effect is even smaller $(\approx 0.1 \mathrm{eV})$ for the $a_{g} \rightarrow b_{3 u}$ transition of the $M^{+}$center.

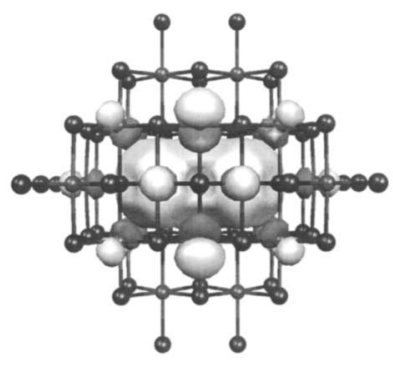

(a)

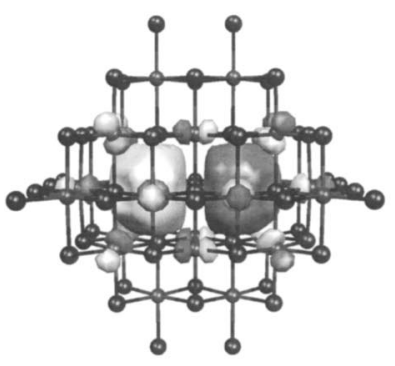

(b)

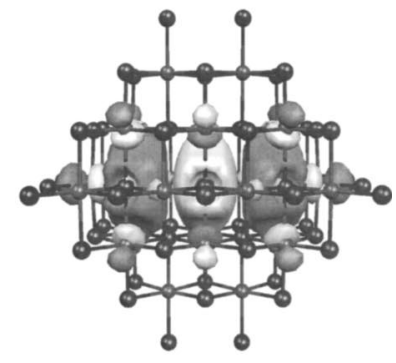

(c)
FIG. 2. Contour plots of the molecular orbitals corresponding to the energy levels associated to a bulk $M$ center. (a) doubly occupied $1 a_{g}$ orbital, (b) doubly $(M)$ or singly $\left(M^{+}\right)$occupied $b_{3 u}$ orbital, (c) virtual $2 a_{g}$ orbital. The shape of the orbitals is derived from a DFT-B3LYP calculation on the ground state of the $M$ center. 
TABLE IV. Electronic transition energies (in eV) for bulk $M$ and $M^{+}$centers computed at the CASPT2 and TD-DFT (B3LYP) levels at the optimized geometry (in parenthesis oscillator strength). Only spin allowed transitions are reported. Symmetry of the electronic states involved is indicated.

\begin{tabular}{lccc}
\hline \hline & $\begin{array}{c}\mathrm{O}_{26} \mathrm{Mg}_{10} \\
\text { CASPT2 (basis D) }\end{array}$ & TD-DFT & $\begin{array}{c}\mathrm{O}_{22} \mathrm{Mg}_{24} \\
\text { CASPT2(basis D) }\end{array}$ \\
\hline Bulk $M$ & & & \\
${ }^{1} A_{g} \rightarrow{ }^{1} B_{3 u}$ & $4.45(0.839)$ & $4.36(0.756)$ & 4.36 \\
\hline Bulk $M^{+}$ & & $1.50(0.071)$ & 1.27 \\
${ }^{2} B_{3 u} \rightarrow 1^{2} A_{g}$ & $1.26(0.062)$ & $3.99(0.450)$ & 3.71 \\
${ }^{2} B_{3 u} \rightarrow 2^{2} A_{g}$ & $3.76(0.531)$ & & \\
\hline \hline
\end{tabular}

Let us compare now the electronic transitions in the bulk as obtained from CASPT2 and TD-DFT (B3LYP) results, Table IV. For the neutral $M$ center, both CASPT2 and TDDFT indicate a very intense ${ }^{1} A_{g} \rightarrow{ }^{1} B_{3 u}$ allowed transition at about $4.4 \mathrm{eV}$. For $M^{+}$a transition with strong intensity is found at $3.7-4.0 \mathrm{eV}$; this transition has the same character as for the $M$ center, i.e., it involves the excitation from the singly occupied $b_{3 u}$ level to the empty $a_{g}$ one. As we mentioned before, another, not very intense, transition is found at $1.3-1.5 \mathrm{eV}$ corresponding to the excitation of one electron from the doubly occupied $a_{g}$ level to the singly occupied $b_{3 u}$ one. Therefore, there is a substantial agreement between CASPT2 and TD-DFT results. This can be attributed to the reasonably localized nature of the levels involved, which allows an unambiguous description of the different states. The different behavior of TD-DFT in bulk $F$ and $M$ centers can be attributed to the presence of two cavities in the latter, which reduces the two-electron repulsion in the excited state. This effect plus the energy lowering of the $M$ center levels produced by the coupling of the individual $F$ center levels (cf. Fig. 1) lead to a considerable decrease in the mixing with conduction band levels and hence improving the efficiency of the TD-DFT treatment.

The results obtained do not allow a clear assignment of the computed transitions to observed bands in neutron or electron irradiated $\mathrm{MgO}$ samples. Only the second intense transition of the $M^{+}$center $(3.7-4 \mathrm{eV})$ is close enough to the $3.5 \mathrm{eV}$ band attributed to $F$ centers aggregates. On the other hand, as we have found a tendency to overestimate the optical transitions in $F$ and $F^{+}$centers, we cannot exclude that the lowest excitation of the neutral $M$ centers, located around $4.4 \mathrm{eV}$ according to the CASPT2 calculations, is somewhat overestimated. Still, the difference from the $3.5 \mathrm{eV}$ band appears to be sufficiently large to exclude a firm assignment of this band to a neutral aggregated of two $\mathrm{O}$ vacancies. If this is the result of the aggregation of a larger number of vacancies remains to be clarified.

\section{Surface $M$ and $M^{+}$centers}

The ground states of the surface $M_{S}$ and $M_{S}^{+}$centers have a similar character as for the bulk counterparts. Two electronic levels are associated to the vacancies and are located

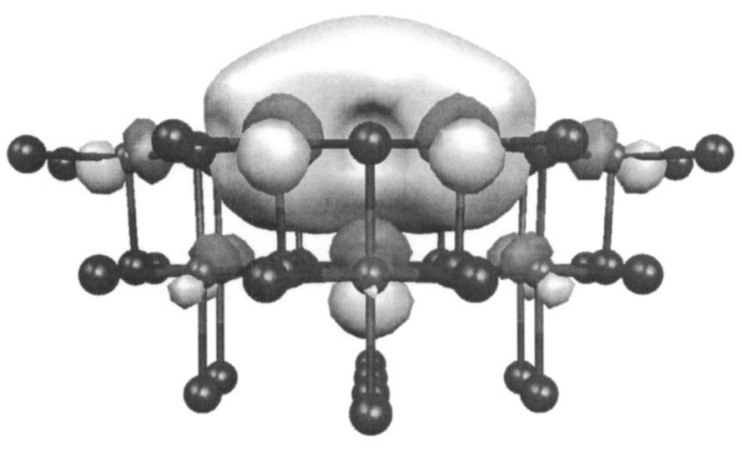

(a)

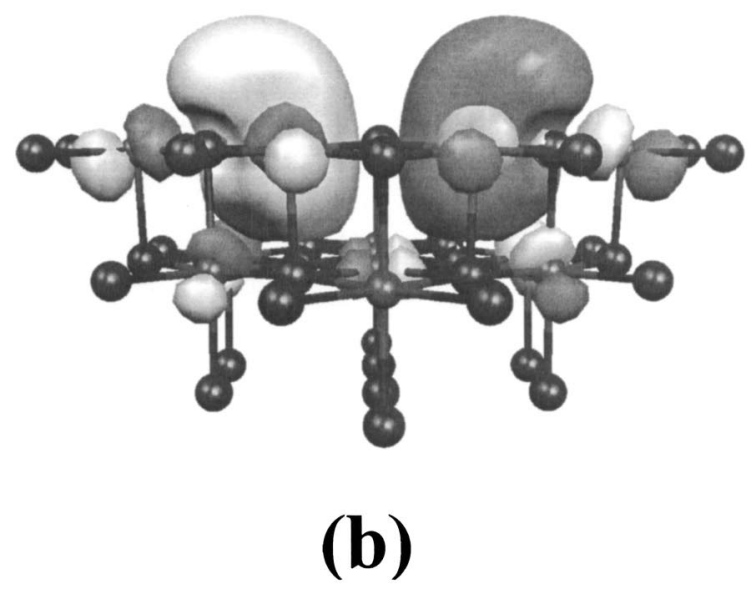

FIG. 3. Contour plots of the molecular orbitals corresponding to the energy levels associated to a surface $M_{S}$ center. (a) doubly occupied $a_{1}$ orbital, (b) doubly $\left(M_{S}\right)$ or singly $\left(M_{S}^{+}\right)$occupied $b_{1}$ orbital. The shape of the orbitals is derived from a DFT-B3LYP calculation on the ground state of the $M$ center.

well below the conduction band ${ }^{20}\left(a_{1}\right.$ and $b_{1}$ in $C_{2 v}$ symmetry, Fig. 3). These levels arise from the bonding and antibonding combinations of the levels of the isolated surface $F_{S}$ centers. In the $M_{S}$ center both levels are doubly occupied and the ground state configuration is ${ }^{1} A_{1}\left(a_{1}\right)^{2}\left(b_{1}\right)^{2}$, whereas in $M_{S}^{+}$the ground state is ${ }^{2} B_{1}\left(a_{1}\right)^{2}\left(b_{1}\right)^{1}$. With respect to the bulk analogs, these electronic levels show an expansion of the electron cloud towards the vacuum, Fig. 3. Empty states in the gap arise from the combination of corresponding empty levels of the isolated $F_{S}$ centers. However, the diffuse nature of the empty levels leads to some mixing with states with conduction band character. A consequence of the expansion of the electron density towards the vacuum is an additional stabilization of the empty levels, especially those arising from the coupling of the two $p_{z}$-type levels of the $F_{s}$ centers (the $z$ axis is normal to the surface plane). This stabilization is a consequence of the reduced electronic repulsion within the cavity, a mechanism which is expected to lower the excitation energies with respect to the bulk. ${ }^{13,14} \mathrm{~A}$ second possible consequence of the "open" nature of the cavity formed by the adjacent $\mathrm{O}$ vacancies on the surface is that the excited levels are not strictly confined in the cavity. 
TABLE V. Electronic transition energies (in eV) for surface $M$ and $M^{+}$centers computed at the CASPT2 and TD-DFT (B3LYP) levels at the optimized geometry (in parenthesis oscillator strength). Only spin allowed transitions are reported. Symmetry of the electronic states involved is indicated.

\begin{tabular}{|c|c|c|c|c|}
\hline & CASPT2 (basis A) & $\begin{array}{c}\mathrm{O}_{14} \mathrm{Mg}_{16} \\
\text { CASPT2 (basis B) }\end{array}$ & TD-DFT & $\begin{array}{c}\mathrm{O}_{18} \mathrm{Mg}_{8} \\
\text { CASPT2 (basis B) }\end{array}$ \\
\hline \multicolumn{5}{|l|}{ Surface $M$} \\
\hline${ }^{1} A_{1} \rightarrow{ }^{1} B_{1}$ & 2.29 & $2.00(0.045)$ & $1.19(0.084)$ & $2.06(0.030)$ \\
\hline${ }^{1} A_{1} \rightarrow{ }^{1} A_{1}$ & 3.28 & $2.95(0.065)$ & $1.48(0.020)$ & $3.01(0.012)$ \\
\hline${ }^{1} A_{1} \rightarrow{ }^{1} B_{2}$ & 3.84 & $3.43(0.180)$ & & $3.54(0.087)$ \\
\hline \multicolumn{5}{|l|}{ Surface $M^{+}$} \\
\hline${ }^{2} B_{1} \rightarrow 1^{2} A_{1}$ & 1.59 & $1.58(0.024)$ & $1.56(0.048)$ & $1.56(0.026)$ \\
\hline${ }^{2} B_{1} \rightarrow 2^{2} A_{1}$ & 2.25 & $2.13(0.002)$ & $1.70(0.009)$ & $2.14\left(4 \times 10^{-4}\right)$ \\
\hline${ }^{2} B_{1} \rightarrow{ }^{2} B_{1}$ & 3.04 & $2.88(0.017)$ & $2.03(0.031)$ & $2.90(0.024)$ \\
\hline${ }^{2} B_{1} \rightarrow{ }^{2} A_{2}$ & 3.43 & $3.27(0.002)$ & & $3.33(0.001)$ \\
\hline
\end{tabular}

For the surface $M_{S}^{+}$center, the lowest allowed transition involves the excitation of one electron from the $\left(a_{1}\right)^{2}$ level to the $\left(b_{1}\right)^{1}$ one, as in the bulk $M^{+}$center. The geometry optimization for the ground state performed at the DFT (B3LYP) level shows a slightly larger relaxation with respect to the bulk case. For the neutral defect the distances between the center of the vacancy and the nearest magnesium atoms expands in average by $2.67 \%$, whereas for the $M_{S}^{+}$center the average expansion is of $4.63 \%$. Differently from the bulk case, we observe a significant effect of the geometry relaxation on the computed excitation energies. The excitation energies for the $M_{S}$ center vary from 0.05 to $0.4 \mathrm{eV}$ whereas those for the $M_{S}^{+}$center vary from 0.4 to $0.7 \mathrm{eV}$ except for the lowest electronic transition for the $M_{S}^{+}$center. This is because of the localized character of this transition which cannot occur in the $M_{S}$ center.

Table V reports CASPT2 results for the two clusters and the two basis sets used showing that the computed transitions are essentially converged with respect to these two variables. The CASPT2 excitations energies are compared with the TD-DFT (B3LYP) results. The lowest allowed transition for the neutral $M_{S}$ center $\left(b_{1} \rightarrow a_{1}\right)$ predicted by CASPT2 occurs at around $2.0 \mathrm{eV}$. For $M_{S}^{+}$the lowest excitation energy $\left(a_{1} \rightarrow b_{1}\right)$ occurs at $1.56 \mathrm{eV}$. TD-DFT gives a lower value for the first transition of the $M_{S}$ center $1.19 \mathrm{eV}$ and an identical value for the $M_{S}^{+}$center $1.56 \mathrm{eV}$. The origin of the discrepancy for the first transition in the $M_{S}$ center is not easy to explain. This discrepancy is found also for all the other transitions of both the $M_{S}$ and $M_{S}^{+}$centers. Thus, it seems that the identical value provided for the lowest transition of $M_{S}^{+}$ is not the rule. We try to provide an explanation for these results. The lowest transition in $M_{S}^{+}$involves the doubly and singly occupied vacancy levels $a_{1}$ and $b_{1}$. These levels are both occupied and hence optimized in the Kohn-Sham variational procedure leading to reasonably localized states. On the other hand, the empty levels associated to the vacancy are again close to the conduction band orbitals, and are partially mixed with these states. One could argue that the more diffuse character of the empty levels involved in those transitions is the reason for the different transition energies in
CASPT2 and TD-DFT values. As we mentioned before, this is not the case for the lowest transition of $M_{S}^{+}$where only the $a_{1}$ and $b_{1}$ levels, well below the conduction band edge, are involved.

\section{CONCLUSIONS}

In this study we have considered the electronic structure of neutral and charged $M$ centers in the bulk and on the surface of $\mathrm{MgO}$ and we have computed the corresponding electronic transitions in order to provide a firm basis for the interpretation of measurements recently done on $\mathrm{MgO}$ thin films. The calculations have been done using two computational approaches: the wave function based CASSCF/ CASPT2 method where correlation effects and multiconfigurational nature of the wave function are explicitly taken into account and the TD-DFT approach. This latter method is much less tested in the context of the optical properties of localized defects. The ground state optimal geometry of the defects considered in this work has been determined at the HF and DFT-B3LYP level making use of cluster of ions embedded in a polarizable environment (shell model approach).

The results can be summarized as follows: The CASPT2 excitation energies for the neutral and charged $F$ centers have been computed with an error of $\sim 1$ and $\sim 5 \%$, respectively, Table VI. Similar errors can be expected when the same computational method is applied to the study of the $M$ centers. In the bulk, the $M$ center gives rise to a first allowed transition around $4.4 \mathrm{eV}$. This is about $1 \mathrm{eV}$ larger than a band reported in neutron irradiated samples and attributed to aggregates of $F$ centers. ${ }^{3}$ It should be noted that the origin of this band is not very clear and that this has not been observed by some authors in electron or UV irradiated or additively colored samples. ${ }^{3,8}$ The $M^{+}$bulk defect gives rise to a lower transition at about $1.3 \mathrm{eV}$. This is due to the different nature of the excitations in $M$ and $M^{+}$centers. In $M$ centers the transitions involve the highest doubly occupied level associated to the vacancy and the corresponding empty states below the conduction band arising from the linear combination of empty states of the isolated oxygen vacancies. In $\mathrm{M}^{+}$ centers the first allowed transition is from the doubly occu- 
TABLE VI. Summary of transition energies (in eV) for bulk and surface $F, F^{+}, M, M^{+}$centers computed at CASPT2 and TD-DFT (B3LYP) levels. The results are compared with observed optical bands in $\mathrm{MgO}$. Firm and tentative assignments are proposed. (From similar calculations and models (Ref. 14), values of 3.39 (3.56), 2.92 (2.60), and 2.60 (2.43) eV have been reported for the lowest allowed transitions of $F$ (and $F^{+}$) terrace, step and corner center. Comparison to experiment for the bulk $F$ center has suggested to scale down these transitions energies. Kramer et al. (Ref. 12) have used these scaled values to assign some of the observed transitions.)

\begin{tabular}{ccccc}
\hline \hline & Transition & CASPT2 & TD-DFT & Exp. assignment \\
\hline$F$ center bulk & ${ }^{1} A_{1 g} \rightarrow{ }^{1} T_{1 u}$ & 5.01 & & 5.03 (Ref. 2) (firm) \\
$F^{+}$center bulk & ${ }^{2} A_{1 g} \rightarrow{ }^{2} T_{1 u}$ & 5.22 & & 4.96 (Ref. 2) (firm) \\
$M$ center bulk & ${ }^{1} A_{g} \rightarrow{ }^{1} B_{3 u}$ & 4.45 & 4.36 & \\
$M^{+}$center bulk & ${ }^{2} B_{3 u} \rightarrow 1^{2} A_{g}$ & 1.26 & 1.50 & $1-1.2$ (Refs. 3, 8) (tentative) \\
& ${ }^{2} B_{3 u} \rightarrow 2^{2} A_{g}$ & 3.76 & 3.99 & 3.5 (Refs. 3, 6, 8) (tentative) \\
$M$ center surface & ${ }^{1} A_{1} \rightarrow{ }^{1} B_{1}$ & 2.00 & 1.19 & \\
$M^{+}$center surface & ${ }^{2} B_{1} \rightarrow{ }^{2} A_{1}$ & 1.58 & 1.56 & $1-1.3$ (Refs. 11, 12) (tentative) \\
\hline \hline
\end{tabular}

pied level in the gap to the singly occupied one. A second intense transition for $\mathrm{M}^{+}$centers is computed at $3.7-4.0 \mathrm{eV}$, and involves the same levels as for the $M$ center. This transition could explain the observed band at around $3.5 \mathrm{eV}$ for neutron irradiated samples, Table VI.

On the surface the nature of the transitions for $M_{S}$ and $M_{S}^{+}$centers is the same as in the bulk, with the only difference that the electron density of the ground, and in particular of the excited states can expand towards the vacuum with no geometrical constraint. As a consequence, the corresponding transitions involving empty states occur at much lower energy. This is the case of the $M_{S}$ center which exhibits a lowest transition at $2 \mathrm{eV}$ at the CASPT2 level. The lowest transition in $M_{S}^{+}$, with quite the same character as in the bulk, occurs at a similar energy $1.6 \mathrm{eV}$. This transition could be the origin of a band at $1-1.3 \mathrm{eV}$ observed in electron irradiated $\mathrm{MgO}$ films and tentatively attributed to $M$ centers. ${ }^{12}$ In most cases similar transition energies have been obtained from CASPT2 and TD-DFT approaches. To some extent this reflects the rather localized nature of the electronic states involved. On the other hand, as compared to the
CASPT2 values for the electronic transitions the rather erratic behavior of the TD-DFT indicates that this approach needs to be carefully used when applied to the study of this kind of systems.

\section{ACKNOWLEDGMENTS}

This research has been supported by the Spanish MCyT Grant No. BQU2002-04029-C02-01, by the Italian PRA project ISADORA of the INFM and, in part, by Generalitat de Catalunya Grant No. 2001SGR-00043. Part of the computer time was provided by the Centre de Supercomputació de Catalunya, CESCA, and Centre Europeu de Paral.lelisme de Barcelona, CEPBA, through a grant from the Universitat de Barcelona. D. D.-A. is grateful for the support given by the European Community under Contract No. HPMT-CT2001-00242, and the Generalitat de Catalunya. D.R. is grateful to the European Community for financing his stay in Barcelona through the IHP program under Contract No. HPRI-CT-1999-00071 held by the CESCA/CEBPA. Finally, we thank P. Sushko and A. Shluger for making the GUESS code available.
*E-mail address: f.illas@qf.ub.es

${ }^{1}$ G. Pacchioni, in The Chemical Physics of Solid Surfaces-Oxide Surfaces, edited by D. P. Woodruff (Elsevier, Amsterdam, 2001), Vol. 9.

${ }^{2}$ L. A. Kappers, R. L. Kroes, and E. B. Hensley, Phys. Rev. B 1, 4151 (1970).

${ }^{3}$ Y. Chen, R. T. Williams, and W. A. Sibley, Phys. Rev. 182, 960 (1968).

${ }^{4}$ M. A. Monge, A. I. Popov, C. Ballesteros, R. Gonzalez, Y. Chen, and E. A. Kotomin, Phys. Rev. B 62, 9299 (2000).

${ }^{5}$ V. N. Kuzovkov, A. I. Popov, E. A. Kotomin, M. A. Monge, R. Gonzalez, and Y. Chen, Phys. Rev. B 64, 064102 (2001).

${ }^{6}$ H. Weber, Z. Phys. 130, 392 (1951).

${ }^{7}$ J. P. Molnar and C. D. Hartman, Phys. Rev. 79, 1015 (1950).

${ }^{8}$ H. R. Day, Phys. Rev. 91, 822 (1953).

${ }^{9}$ V. E. Henrich, G. Dresselhaus, and H. J. Zeiger, Phys. Rev. B 22, 4764 (1980).
${ }^{10}$ P. R. Underhill and T. E. Gallon, Solid State Commun. 43, 9 (1982).

${ }^{11}$ M-C. Wu, C. M. Truong and D. W. Goodman, Phys. Rev. B 46, 12688 (1992).

${ }^{12}$ J. Kramer, W. Ernst, C. Tegenkamp, and H. Pfnür, Surf. Sci. 517, 87 (2002).

${ }^{13}$ F. Illas and G. Pacchioni, J. Chem. Phys. 108, 7835 (1998).

${ }^{14}$ C. Sousa, G. Pacchioni, and F. Illas, Surf. Sci. 429, 217 (1999).

${ }^{15}$ A. M. Ferrari and G. Pacchioni, J. Phys. Chem. 99, 17010 (1995).

${ }^{16}$ A. M. Ferrari and G. Pacchioni, Int. J. Quantum Chem. 58, 241 (1996).

${ }^{17}$ G. Pacchioni, A. M. Ferrari, and G. Ieranò, Faraday Discuss. 106, 155 (1997).

${ }^{18}$ E. Giamello, M. C. Paganini, D. M. Murphy, A. M. Ferrari, and G. Pacchioni, J. Phys. Chem. 101, 971 (1997).

${ }^{19}$ G. Pacchioni and P. Pescarmona, Surf. Sci. 412/413, 657 (1998). 
${ }^{20}$ F. Finocchi, J. Goniakowski, and C. Noguera, Phys. Rev. B 59, 5178 (1999).

${ }^{21}$ E. Miyoshi, Y. Miyake, S. Katsuki, and Y. Sakai, J. Mol. Struct.: THEOCHEM 451, 81 (1998).

${ }^{22}$ C. Sousa and F. Illas, J. Chem. Phys. 115, 1435 (2001).

${ }^{23}$ K. Andersson, P.-Å. Malmqvist, B. O. Roos, A. J. Sadlej, and K. Wolinski, J. Phys. Chem. 94, 5483 (1990).

${ }^{24}$ K. Andersson, P.-A. Malmqvist, and B. O. Roos, J. Chem. Phys. 96, 1218 (1992).

${ }^{25}$ M. Petersilka, U. J. Gossmann, and E. K. U. Gross, Phys. Rev. Lett. 76, 1212 (1996).

${ }^{26}$ M. E. Casida, C. Jamorki, K. C. Casida, and D. R. Salahub, J. Chem. Phys. 108, 4439 (1998).

${ }^{27}$ R. E. Stratmann, G. Scuseria, and M. Frisch, J. Chem. Phys. 109, 8218 (1998).

${ }^{28}$ A. Görling, H. H. Heinze, S. P. Ruzankin, M. Staufer, and N. Rösch, J. Chem. Phys. 110, 2785 (1999).

${ }^{29}$ K. Raghavachari, D. Ricci, and G. Pacchioni, J. Chem. Phys. 116, 825 (2002).

${ }^{30}$ B. O. Roos, K. Andersson, M. P. Fülscher, P.- $\AA$. Malmqvist, L. Serrano-Andrés, K. Pierloot, and M. Merchán, Adv. Chem. Phys. 93, 219 (1996).

${ }^{31}$ B. O. Roos, K. Andersson, M. P. Fülscher, L. Serrano-Andrés, K. Pierloot, M. Merchán, and V. Molina, J. Mol. Struct.: THEOCHEM 388, 257 (1996).

${ }^{32}$ A. Bernhardsson, N. Forsberg, P.- $\AA$. Malmqvist, and B. O. Roos, J. Chem. Phys. 112, 2798 (2000).

${ }^{33}$ V. Molina, M. Merchán, B. O. Roos, and P.-Å. Malmqvist, Chem. Phys. 2, 2211 (2000).

${ }^{34}$ C. de Graaf, R. Broer, and W. C. Nieuwpoort, Chem. Phys. 208, 35 (1996)

${ }^{35}$ C. de Graaf and R. Broer, Phys. Rev. B 62, 702 (2000).

${ }^{36}$ C. Sousa, C. de Graaf, and G. Pacchioni, J. Chem. Phys. 114, 6259 (2001).

${ }^{37}$ C. Sousa, C. de Graaf, F. Illas, M. T. Barriuso, J. A. Aramburu, and M. Moreno, Phys. Rev. B 62, 13366 (2000).

${ }^{38}$ K. Andersson, M. Barysz, A. Bernhardsson, M. R. a. Blomberg, D. L. Cooper, T. Fleig, M. P. Fülscher, C. de Graaf, B. A. Hess, G. Karlström, R. Lindh, P.-A. Malmqvist, P. Neográdi, J. Olsen, B. O. Roos, B. Schimmelpfennig, M. Schütz, L. Seijo, L. Serrano-Andrés, P. E. M. Siegbahn, J. Starling, T. Thorsteinsson, V. Veryazov, and P.-O. Widmark, MOLCAS version 5.2, University of Lund, Sweden, 2000.
${ }^{39}$ A. D. Becke, J. Chem. Phys. 98, 5648 (1993).

${ }^{40}$ C. Lee, W. Yang, and R. G. Parr, Phys. Rev. B 37, 785 (1988).

${ }^{41}$ R. Colle and O. Salvetti, Theor. Chim. Acta 37, 329 (1975); 53, 55 (1979).

${ }^{42}$ R. Colle and O. Salvetti, J. Chem. Phys. 79, 1404 (1993).

${ }^{43}$ S. Huzinaga, L. Seijo, Z. Barandiaran, and M. Klobukowski, J. Chem. Phys. 86, 2132 (1987).

${ }^{44}$ J. L. Pascual, L. Seijo, and Z. Barandiaran, J. Chem. Phys. 98, 9715 (1993).

${ }^{45}$ M. A. Nygren, L. G. M. Pettersson, Z. Barandiaran, and L. Seijo, J. Chem. Phys. 100, 2010 (1994).

${ }^{46}$ P. V. Susko, A. L. Shluger, and C. R. A. Catlow, Surf. Sci. 450, 153 (2000).

${ }^{47}$ P. V. Sushko, A. L. Shluger, R. C. Baetzold, and C. R. A. Catlow, J. Phys.: Condens. Matter 12, 8257 (2000).

${ }^{48}$ W. Stevens, H. Bach, and J. Krauss, J. Chem. Phys. 81, 6026 (1984).

${ }^{49}$ M. J. Frisch et al., GAUSSIAN 98, Revision A.6 (Gaussian Inc., Pittsburgh, PA, 1998).

${ }^{50}$ J. E. Dennis and R. Sabel, Numerical Methods for Unconstrained Optimization and Nonlinear Equations (Prentice-Hall, Englewood Cliffs, NJ, 1983).

${ }^{51}$ M. M. Francl, W. J. Petro, W. J. Hehre, J. S. Binkley, M. S. Gordon, D. J. DeFrees, and J. A. Pople, J. Chem. Phys. 77, 3654 (1982).

${ }^{52}$ W. J. Hehre, R. Ditchfield, and J. A. Pople, J. Chem. Phys. 56, 2257 (1972).

${ }^{53}$ P. C. Hariharan and J. A. Pople, Theor. Chim. Acta 28, 213 (1973).

${ }^{54}$ T. Clark, J. Chandrasekhar, G. W. Spitznagel, and P. v. R. Schleyer, J. Comput. Chem. 4, 294 (1983).

${ }^{55}$ P.-O. Widmark, P.-A. Malmqvist, and B. O. Roos, Theor. Chim. Acta 77, 291 (1990).

${ }^{56}$ P.-O. Widmark, B. J. Persson, and B. O. Roos, Theor. Chim. Acta 79, 419 (1991).

${ }^{57}$ I. de P. R. Moreira, F. Illas, and R. L. Martin, Phys. Rev. B 65, 155102 (2002).

${ }^{58}$ J. Muscat, A. Wander, and N. M. Harrison, Chem. Phys. Lett. 342, 397 (2001).

${ }^{59}$ P. S. Bagus, F. Illas, and C. Sousa, J. Chem. Phys. 100, 2943 (1993).

${ }^{60}$ C. Kölmel and C. S. Ewig, J. Phys. Chem. B 105, 8538 (2001). 